\title{
Varroosis en abejas melíferas en diferentes condiciones ambientales y regionales de Jalisco, México
}

\section{Varroosis in honey bees in different environmental and regional conditions of Jalisco, Mexico}

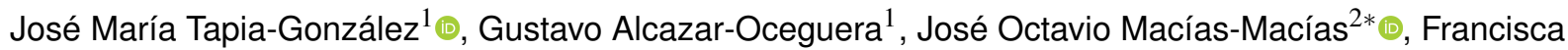

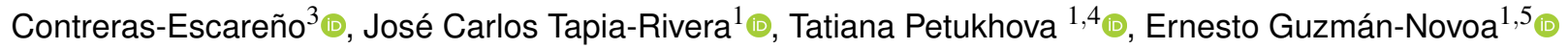 \\ ${ }^{1}$ Departamento de Ciencias Económicas y Administrativas. Centro Universitario del Sur. Universidad de Guadalajara. Av. Enrique \\ Arreola Silva 883. CP. 49000. Cd. Guzmán, Jalisco. México. \\ ${ }^{2}$ Departamento de Ciencias de la Naturaleza. Centro Universitario del Sur. Universidad de Guadalajara. Av. Enrique Arreola Silva 883. \\ CP. 49000. Cd. Guzmán, Jalisco. México. \\ ${ }^{3}$ Departamento de Producción Agrícola. Centro Universitario de la Costa Sur. Avenida Independencia Nacional 161. CP. 48900. Autlán \\ de Navarro, Jalisco, México. \\ ${ }^{4}$ Department of Population Medicine, OVC, University of Guelph, 50 Stone Road East, Guelph, N1G 2W1, Ontario, Canada. \\ ${ }^{5}$ School of Environmental Sciences, University of Guelph, 50 Stone Road East, Guelph, N1G 2W1, Ontario, Canada. \\ *Autor de correspondencia: joseoc@cusur.udg.mx
}

Artículo científico recibido: 17 de octubre de 2018 aceptado: 14 de diciembre de 2018

RESUMEN. El objetivo fue determinar la prevalencia de Varroa destructor, el grado de infestación del ácaro y la relación del parásito con variables geográficas y climáticas en 369 colonias de abejas Apis mellifera de nueve municipios de zonas templadas y cálidas del estado de Jalisco, México. Se encontró una prevalencia promedio de $88 \%$ y nivel de infestación de $5.2 \%$ del parásito, con diferencias significativas entre municipios para estos parámetros $(p<0.05)$, pero sin diferencias entre zonas climáticas. La prevalencia de $V$. destructor fue alta en algunos municipios, con el promedio de muestras positivas del $100 \%$ y porcentajes de infestación de más del $8 \%$. El nivel promedio de infestación del $5.2 \%$ fue similar al que recomienda la SAGARPA como tolerable en México (5\%), sin embargo, más de la tercera parte de la colonias rebasaban estos niveles y algunas tenían más de 35\% de infestación. Los factores climáticos analizados y la altura sobre el nivel del mar no tuvieron una relación significativa con la prevalencia y niveles de parasitismo de $V$. destructor. Esto se puede deber a la alta variabilidad de los datos, lo que sugiere que otros factores influencian en mayor medida la prevalencia y grado de infestación de las colonias de abejas dentro de cada región geográfica. Debido a la alta prevalencia de $V$. destructor se debe considerar realizar estudios posteriores para determinar cuáles son las causas de estos hallazgos, a fin de que se tomen medidas para su control.

Palabras clave: Apis mellifera, infestación, prevalencia, sur de Jalisco, Varroa destructor.

ABSTRACT. The objective of this study was to determine the prevalence of Varroa destructor, the degree of mite infestation and the relationship of the parasite with geographic and climatic variables in 369 colonies of honey bees (Apis mellifera) from nine municipalities of temperate and warm areas from the state of Jalisco, Mexico. An average prevalence of $88 \%$ and an infestation level of $5.2 \%$ of $V$. destructor were found, with significant differences between municipalities for theses parameters $(\mathrm{p}<0.05)$, but with no significant differences between climatic zones. The prevalence of $V$. destructor was particularly high in some municipalities, where the average of positive samples reached $100 \%$, with percentages of infestation of $>8 \%$. The $5.2 \%$ mite infestation level was similar to that recommended by SAGARPA as tolerable in Mexico (5\%), however, more than a third of the colonies exceeded these levels and some had $>35 \%$ infestation. The climatic factors analyzed did not have a significant relationship with the prevalence and levels of $V$. destructor parasitism. This was due to the high variability of the data, which suggests that other factors influence the prevalence and degree of $V$. destructor infestation of honey bee colonies to a greater extent within each geographic region. Similarly, the altitude above sea level did not have significant effects on the prevalence and infestation levels of $V$. destructor in this study. Due to the high prevalence of $V$. destructor in honey bee colonies of southern Jalisco as well as to the high percentage of them having levels of parasitism $>5 \%$, a subsequent study 
should be carried out to determine what are the causes of these findings, in order to take measures for its control.

Key words: Apis mellifera, infestation, prevalence, southern Jalisco, Varroa destructor.

\section{INTRODUCCIÓN}

La infestación por el ácaro Varroa destructor ocasiona la varroosis en abejas melíferas (Apis mellifera L.) (Anderson y Trueman 2000), la cual está distribuida en la mayoría de los países del mundo (Nazzi y Le Conte 2016). En México, la varroosis fue reportada por primera vez en 1992 en colonias de abejas del estado de Veracruz (Chihu et al. 1992); desde entonces, se ha dispersado a todas las entidades de la república mexicana (Guzman-Novoa et al. 2012). El acaro $V$. destructor es un parásito externo que se alimenta de la hemolinfa y tejido graso de las abejas adultas y de sus crías, reproduciéndose en estas últimas, lo que afecta severamente la salud de las abejas (Boecking y Genersch 2008). Este acaro es la mayor amenaza sanitaria de las abejas melíferas y una de las razones principales de la muerte y despoblamiento de colonias en el mundo, ocasionando pérdidas económicas mayores que las causadas por el resto de las enfermedades apícolas conocidas (Boecking y Genersch 2008, GuzmánNovoa et al. 2010, Nazzi y Le Conte 2016). Se ha detectado a $V$. destructor en el $98 \%$ de las colonias afectadas por el síndrome del colapso de la colonia (SCC) y es un factor significativo correlacionado con este problema (Guzman-Novoa 2016). En México, anualmente se pierde más del $33 \%$ de las colonias de abejas de las regiones del altiplano y norte del país, atribuidas en gran medida a parasitosis causadas por V. destructor (Medina-Flores et al. 2018). Las infestaciones por $V$. destructor inhiben el sistema inmune de las abejas haciéndolas más propensas a otros patógenos y factores estresantes como los insecticidas (Koleoglu et al. 2017, 2018; Tarek et al. 2018). Además, hay evidencias de que $V$. destructor trasmite varios virus a las abejas, entre los que se encuentran el virus de las alas deformes, de la parálisis crónica y de la parálisis aguda israelí (SantillánGalicia et al. 2010, Guzman-Novoa et al. 2012). A pesar de que las abejas africanizadas son relativamente más resistentes a la parasitosis por $V$. destruc- tor que abejas de razas europeas (Guzman-Novoa et al. 2011), en México, se ha demostrado que infestaciones medianas y altas de $V$. destructor, disminuyen la producción de miel de colonias de abejas africanizadas (Arechavaleta-Velasco y GuzmanNovoa 2000, Medina-Flores et al. 2011).

Los daños causados por $V$. destructor a las abejas son difíciles de atenuar, debido a que la resistencia del parásito a los acaricidas usados para controlarlo se ha incrementado y aunque se hacen rotaciones en el uso de estos productos, cada vez se requiere una mayor cantidad de ellos para controlar al ácaro (Mitton et al. 2018). Además, algunos estudios han encontrado que factores climáticos como la temperatura y la humedad, influyen en la infestación de $V$. destructor, y en la mortalidad que la parasitosis causa a las abejas (Meixner et al. 2015, Hou et al. 2016). Sin embargo, en la actualidad se conoce poco sobre la situación de la varroosis y las relaciones que puede tener con variables geográficas y climáticas en apiarios de México. Por ello, el objetivo del presente estudio fue determinar la prevalencia de $V$. destructor, el porcentaje de infestación del ácaro y la relación del parásito con variables geográficas y climáticas en colonias de abejas melíferas de municipios de la región sur-sureste del estado de Jalisco, México.

\section{MATERIALES Y MÉTODOS}

El presente estudio se realizó en nueve municipios ubicados en dos zonas climáticas consideradas como templada subhúmedo y cálida subhúmedo, ubicados en el occidente de México ( $19^{\circ} 24^{\prime}, 21^{\circ} 14^{\prime}$ LN; $101^{\circ} 59^{\prime}, 104^{\circ} 5^{\prime}$ LO) e incluyen a Zapotlán el Grande, Gómez Farías y Tapalpa, con clima templado subhúmedo y los municipios de Tecalitlán, Tamazula, Unión de Guadalupe, Zacoalco de Torres, Sayula, y Cocula, con clima cálido subhúmedo (Gobierno del Estado de Jalisco 2012, INEGI 2013). Los municipios con clima cálido subhúmedo tienen una temperatura media anual de $21{ }^{\circ} \mathrm{C}$ sin cambio térmico invernal 
bien definido (INEGI 2015). El régimen de lluvias se registra de junio a septiembre, con una precipitación promedio de $801 \mathrm{~mm}$ y una altitud promedio de 1 495 msnm (INEGI 2013). Los municipios con clima templado subhúmedo presentan un cambio térmico invernal bien definido, temperatura media anual de $14.4{ }^{\circ} \mathrm{C}$ y precipitación media anual de $1117 \mathrm{~mm}$; la altitud promedio es de 1711 msnm (INEGI 2015). En las dos zonas geográficas se tomaron al azar entre marzo y mayo del 2016, muestras de 369 colonias de abejas melíferas ubicadas en 143 apiarios. El tamaño de la muestra se calculó para aproximadamente un $5 \%$ de las colmenas existentes en los municipios evaluados; de las que se realizó un muestreo aleatorio estratificado (Tabla 1). Se utilizó un método cuantitativo para detectar la presencia de $V$. destructor en abejas adultas de cada colonia, así como su grado de infestación. De cada colmena a evaluar se colectaron aproximadamente 300 abejas tomadas de un panal del nido de cría, las que se depositaron en un envase de plástico de boca ancha ( $500 \mathrm{~mL}$ ), el cuál contenía $200 \mathrm{~mL}$ de etanol al $70 \%$. Las muestras se evaluaron posteriormente en el laboratorio de microbiología del Centro Universitario del Sur de la Universidad de Guadalajara de acuerdo con la metodología de De Jong et al. (1982). Cada envase se agitó durante 1 min con el fin de que los ácaros se desprendieran de las abejas. Posteriormente, se vació el contenido del envase con las abejas en una coladera que permitió el paso de los ácaros a través de una malla cribada (cuando la muestra era positiva), para ser recolectados en una tela blanca. Los ácaros y las abejas se contaron y se determinó el promedio de infestación de cada muestra positiva. Posteriormente se dividió el número de ácaros obtenidos entre el número de abejas por muestra y después se multiplicó el resultado por 100. En cada apiario muestreado se registró la altitud sobre el nivel del mar (msnm), con un GPS (Sportrack-color, Magellan). Además de los datos de temperatura ambiental $\left({ }^{\circ} \mathrm{C}\right)$ y precipitación pluvial (mm) obtenidos de la Comisión Nacional del Agua (CONAGUA 2015). En los análisis estadísticos, las variables de respuesta fueron el número de colonias infestadas y el porcentaje de infestación con el ácaro $V$. destructor en abejas adultas entre diferentes mu- nicipios y zonas, para determinar prevalencia y grados de infestación del ácaro, respectivamente. Los predictores (variables explicativas) incluidos en los análisis fueron, temperatura ambiental, precipitación pluvial y altura sobre el nivel del mar (msnm). Los análisis estadísticos se efectuaron con las siguientes técnicas: la prevalencia de varroosis (porcentaje de colonias infestadas) entre zonas y entre municipios se analizó mediante comparaciones múltiples por pares, cuyas probabilidades fueron ajustadas con el método de Benjamini y Hochberg. Para analizar el grado promedio de infestación de las colonias, la suposición de normalidad se verificó con la prueba de ShapiroWilk y el supuesto de homogeneidad se comprobó con la prueba de Bartlett, siendo ambas suposiciones no satisfactorias. Por ello, los municipios y zonas se compararon con respecto al nivel de infestación de las colonias con $V$. destructor, mediante las pruebas no paramétricas de Kruskal-Wallis y Mann-Whitney $\mathrm{U}$, respectivamente, ya que los datos no pudieron normalizarse. Las medias se separaron mediante comparaciones múltiples por pares con la prueba de Dunn, ya que el número de observaciones por municipio eran desiguales. Además, con el objeto de evaluar la asociación entre las variables climáticas (temperatura y precipitación pluvial) y la variable geográfica $(\mathrm{msnm})$ con la prevalencia y grado de infestación de varroosis de las colonias, se realizaron análisis de correlación de Kendall. Para los análisis estadísticos se empleó la versión R 3.3.1 con un nivel de significancia de $p<0.05$.

\section{RESULTADOS}

La prevalencia de varroosis en las colonias de abejas de los nueve municipios incluidos se muestra en la Tabla 1. De 369 muestras evaluadas, $V$. destructor se detectó en 324 (87.8\%), con rango de 54 a 100\%, encontrándose diferencias significativas en la prevalencia de la parasitosis entre municipios ( $p<0.05$ ). El $88.7 \%$ de las colonias de la zona cálida subhúmeda estaban parasitadas con $\mathrm{V}$. destructor, mientras que en la templada subhúmeda, el $84.1 \%$ tuvieron la presencia del acaro. Pero no se encontró diferencia significativa en la prevalencia 
Tabla 1. Número de apiarios, colonias muestreadas y prevalencia de Varroa destructor por municipio y zona climática, en el sur de Jalisco.

\begin{tabular}{llllll}
\hline Municipio & Apiarios & Colonias & Zona climática & Colonias positivas & Prevalencia (\%) \\
\hline Zapotlán el Grande & 10 & 24 & Templada & 21 & $87.50^{b}$ \\
Gómez Farías & 6 & 15 & Templada & 9 & $60.00^{c}$ \\
Tapalpa & 12 & 30 & Templada & 28 & $93.33^{a, b}$ \\
Cocula & 16 & 41 & Cálida & 22 & $53.66^{c}$ \\
Tamazula & 36 & 93 & Cálida & 93 & $100.00^{a}$ \\
Sayula & 20 & 53 & Cálida & 50 & $94.34^{a, b}$ \\
Unión de Gpe. & 8 & 20 & Cálida & 20 & $100.00^{a}$ \\
Tecalitlán & 21 & 54 & Cálida & 47 & $87.04^{b}$ \\
Zacoalco de Torres & 14 & 39 & Cálida & 34 & $87.18^{b}$ \\
Total & 143 & 369 & & 324 & 87.80 \\
\hline
\end{tabular}

de la varroosis entre colonias de ambas zonas $\left(\chi^{2}\right.$ $=0.724, \mathrm{gl}=1, \mathrm{p}=0.394)$. El nivel de infestación con $V$. destructor de las colonias de los diferentes municipios se muestra en la Figura 1, con diferencias significativas para esta variable entre municipios (Kruskal-Wallis $\chi=50.96, \mathrm{gl}=8, \mathrm{p}<0.01$ ). Los municipios con los porcentajes de infestación de $V$. destructor por colonia más altos fueron Tecalitlán y Unión de Guadalupe, con $7.4 \pm 0.13$ y $8.3 \pm 0.39 \%$, respectivamente; mientras que los que tuvieron los niveles más bajos, fueron Gómez Farías y Cocula con $2.4 \pm 0.23$ y $2.4 \pm 0.09 \%$, respectivamente. La infestación media de las colonias con $V$. destructor fue de $5.2 \pm 0.14$ en general $y$, de $5.5 \pm 0.12$ y 4.2 $\pm 0.16 \%$ para las zonas cálida y templada, respectivamente, con diferencias no significativas ( $\mathrm{W}=119$, $\mathrm{gl}=368, \mathrm{p}>0.05)$. La distribución del nivel de infestación con $V$. destructor entre las colonias de cada municipio se muestra en la Figura 2, donde se observa que el porcentaje de infestación del ácaro entre colonias de cada municipio fue variable, en particular en los municipios de la zona cálida subhúmeda. Cabe señalar que salvo para los municipios de Tamazula y Unión de Guadalupe, donde todas las colonias estaban infestadas, el nivel mínimo de infestación con el ácaro en los demás municipios fue $0 \%$, mientras que el máximo fue de más del $10 \%$, con colonias que llegaron a tener más del 35\% de infestación (Unión de Guadalupe). Pero más del $50 \%$ de las colonias de siete de los nueve municipios, tienen niveles de infestación menores del 5\%. Solo en Tecalitlán y Unión de Guadalupe, la mayoría de las colonias tienen nive- les de infestación con $V$. destructor de más del $5 \%$. No se encontraron correlaciones significativas entre las variables climáticas (temperatura y precipitación pluvial) y la variable geográfica (msnm) con la prevalencia y grado de infestación de varroosis de las colonias $(p>0.05)$.

\section{DISCUSIÓN}

Este es el primer trabajo que se realiza en el occidente de México para determinar la prevalencia y niveles de infestación del ácaro $V$. destructor en colonias de abejas de municipios ubicados en regiones climáticas diferentes. El $88 \%$ de las colonias están parasitadas con el ácaro, alta prevalencia que concuerda con el $79 \%$ de prevalencia reportado y los niveles de infestación promedio de $V$. destructor por colonia para las zonas cálida y templada, fueron similares a lo reportado para zonas climáticas semicálida y templada (Medina-Flores et al. 2014b). Tanto la prevalencia como el nivel de infestación de $V$. destructor encontrado en este estudio y el de Medina-Flores et al. (2014b) fueron mayores que lo reportado para estos parámetros en estudios realizados en Yucatán, México; que reportan la prevalencia de colonias manejadas de 63 a $68 \%$, con niveles de infestación mayores al 5\% (Medina 1998, Martínez-Puc et al. 2011). Estas diferencias en prevalencia e infestación de $V$. destructor en las abejas entre Yucatán y regiones del altiplano mexicano, podrían explicarse por efectos climáticos y genotípicos. Climas más cálidos y secos, así como mayor 


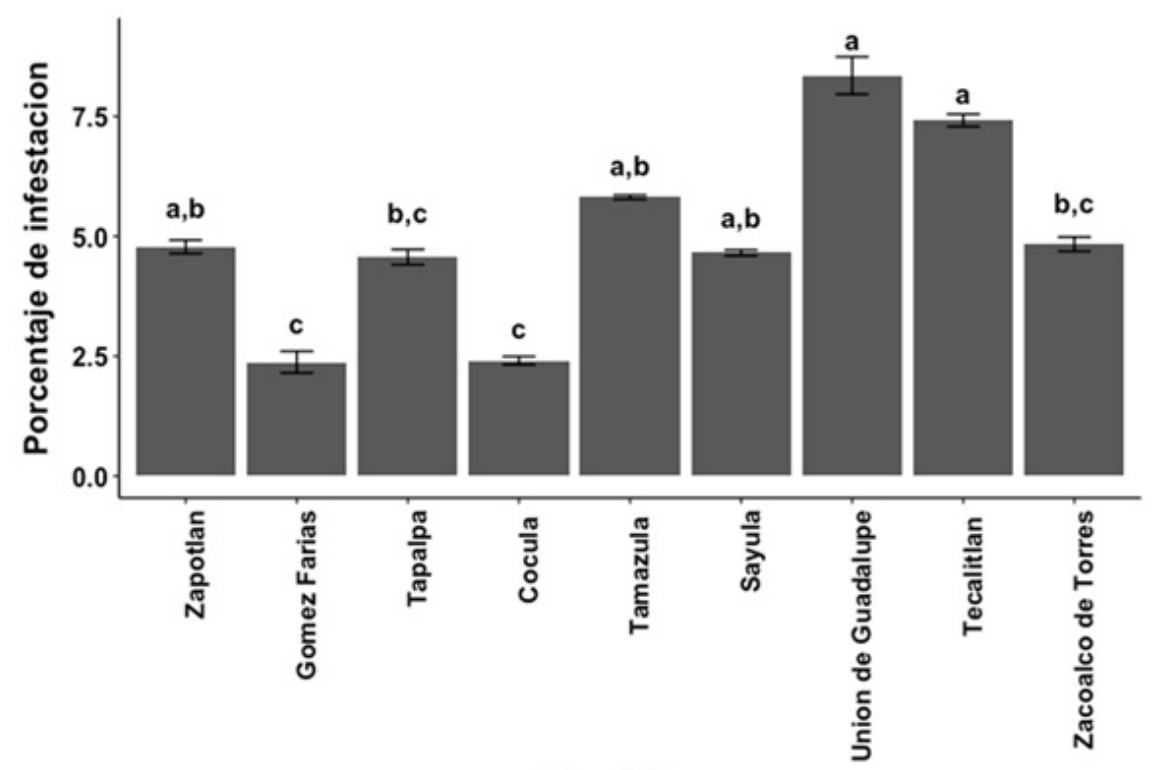

Municipios

Figura 1. Medias \pm errores estándar para el porcentaje de infestación de abejas melíferas adultas de colonias parasitadas con el ácaro Varroa destructor en nueve municipios del sur del estado de Jalisco, México. Literales diferentes sobre las barras, representan diferencias significativas basadas en pruebas de Kruskal-Wallis y de Dunn $(p<0.05)$.

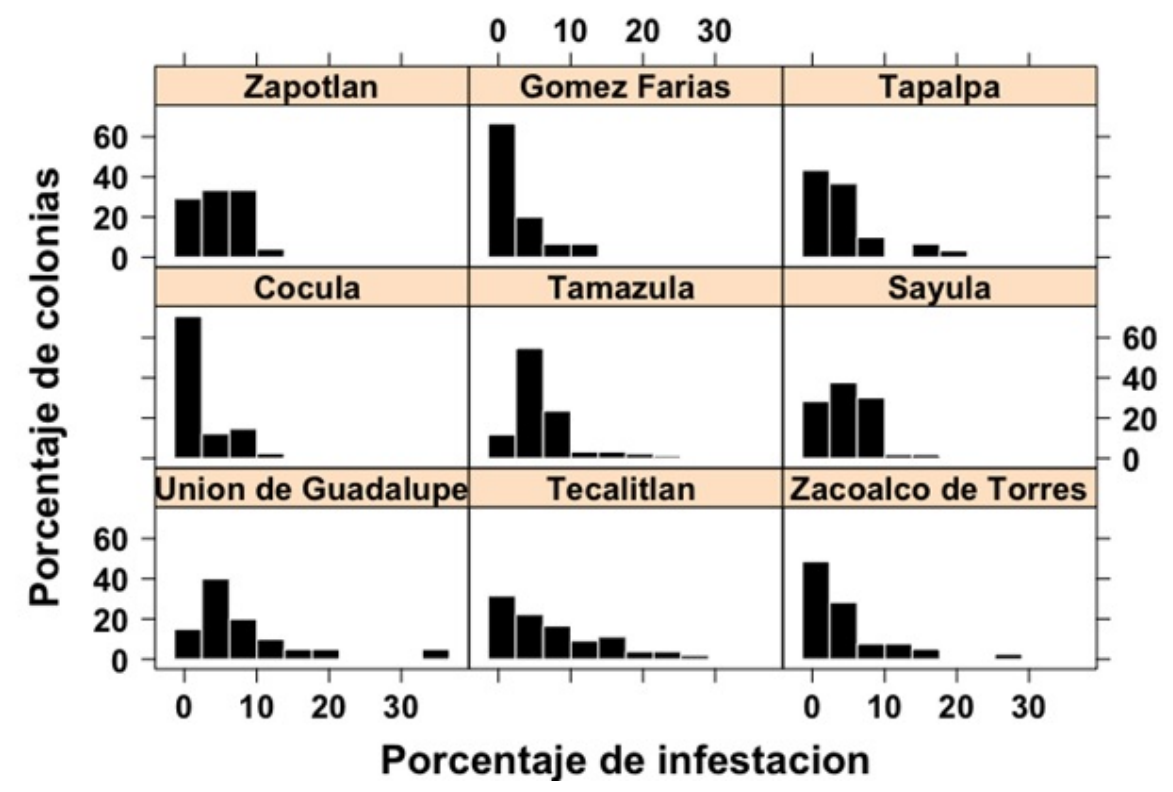

Figura 2. Porcentaje de colonias con diferentes niveles (\%) de infestación por Varroa destructor en colonias de abejas melíferas de nueve municipios de Jalisco, México, durante 2016.

grado de africanización de las abejas, como ocurre en Yucatán, restringen la dispersión y reproducción del ácaro (Medina y Martin 1999, Mondragon et al.
2005, Medina-Flores et al. 2014a). Aunque se encontraron diferencias entre municipios para prevalencia y niveles de infestación del ácaro, no se detec- 
taron diferencias para estos parámetros entre las dos zonas climáticas evaluadas, ni correlaciones significativas entre variables climáticas y geográficas con la prevalencia y grado de parasitismo de la varroosis. Estos resultados son similares a los reportados por Anguiano-Baez et al. (2016), quienes no encontraron prevalencia significativa en el nivel de infestación por $V$. destructor en colonias de clima templado (altiplano mexicano) en comparación con colonias de clima tropical (costa de Nayarit) en México, pero se difiere de los resultados reportados por Meixner et al. (2015), quienes afirman que la infestación de las colonias de abejas con el ácaro está fuertemente influenciada por el clima. En relación al clima, se sabe que las diferencias entre estudios pueden deberse a múltiples variables como el manejo de las colonias, métodos de control de la varroosis, época del año en que se muestrea y genotipo de las abejas (Medina 1998, Harris et al. 2003, Giacobino et al. 2017). Además, se encontró alta variación en la prevalencia y niveles de infestación del ácaro dentro y entre municipios de ambas zonas, lo que probablemente no permitió detectar significancia con las variables climáticas y de altitud. La Secretaría de Agricultura, Ganadería, Desarrollo Rural y Pesca (SAGARPA) indica que el objetivo de la Campaña de Diagnóstico, Prevención y Control de la Varroosis es mantener el nivel de infestación del ácaro $V$. destructor en las colonias de abejas por debajo del 5\%, para atenuar sus daños (SARH 1994). En relación a esta recomendación en la presente investigación el nivel de infestación con $V$. destructor encontrado en colonias de abejas de Jalisco fue de $5.2 \%$, porcentaje similar al que la SAGARPA recomienda como nivel de tolerancia en México. Si se toma en cuenta solo el promedio de infestación encontrado para toda la región sur/sureste de Jalisco, puede concluirse que los apicultores de esta región de México, tienen relativamente controlado al ácaro $V$. destructor, en las colonias manejadas. Pero si se analiza la alta variación en niveles de infestación del ácaro en las colonias dentro y entre los municipios (Figura 2), esa conclusión no es válida para aproximadamente el $39 \%$ de las colonias, particularmente para los munici- pios de Tecalitlán y Unión de Guadalupe, donde más del $50 \%$ de las colonias tiene niveles de infestación mayores al $5 \%$. Esta alta variación de infestación del ácaro entre municipios y apiarios, también fue observada en un estudio realizado en el este del estado de México, el que detectó un grado de parasitosis entre 0.5 y 22\% (Martínez-Cesareo et al. 2016). El hecho de que un alto porcentaje de colonias tenga niveles de infestación mayores al $5 \%$, aunado a que más del $87 \%$ de ellas estén parasitadas, es preocupante y amerita investigaciones sobre sus causas, así como programas para aminorar el impacto negativo de $V$. destructor en la salud y productividad de las colonias de abejas melíferas del estado de Jalisco.

\section{CONCLUSIONES}

Se tiene una elevada prevalencia de la parasitosis en colonias de abejas del sur/sureste del estado de Jalisco, con alta presencia en algunos municipios. El nivel promedio de infestación fue similar al recomendado por la SAGARPA como tolerable en México, pero más de la tercera parte de las colonias rebasaron estos niveles. $\mathrm{Ni}$ los factores climáticos analizados o la altura sobre el nivel del mar tuvieron asociación con la prevalencia y niveles de parasitismo, lo que puede deberse a la alta variación de los datos, y sugiere que otros factores influyen en mayor medida la prevalencia y grado de infestación de las colonias de abejas dentro de cada región geográfica. Debido a la alta prevalencia de $V$. destructor y al porcentaje mayor del $5 \%$, deben considerarse estudios posteriores para determinar las causas. Se recomienda que los productores monitoreen de forma periódica los niveles de infestación en sus colonias, para dar tratamientos oportunos que controlen la parasitosis y los daños que esta causa.

\section{AGRADECIMIENTOS}

A la Federación Mexicana de Apicultores (FMA) delegación Jalisco y a las asociaciones de apicultores de los municipios que colaboraron por facilitar la toma de muestras de sus colonias. 


\section{LITERATURA CITADA}

Anderson DL, Trueman JW (2000) Varroa jacobsoni (Acari: Varroidae) is more than one species. Experimental Applied Acarology 24: 165-189.

Anguiano-Baez R, Guzman-Novoa E, Hamiduzzaman M, Espinosa-Montaño LG, Correa-Benítez A (2016) Varroa destructor (Mesostigmata: Varroidae) parasitism and climate differentially influence the prevalence, levels and overt infections of deformed wing virus in honey bees (Hymenoptera: Apidae). Journal of Insect Science 16(1): 44 Doi 10.1093/jisesa/iew029.

Arechavaleta-Velasco M.E, Guzmán-Novoa E (2000) Honey production with fluvalinate treated and untreated honey bee (Apis mellifera L.) colonies in Valle de Bravo, Mexico. Veterinaria México 31: 381-384.

Boecking O, Genersch E (2008) Varroosis the ongoing crisis in beekeeping. Journal of Consumer Protection Food Safety 3: 221-228.

Chihu AD, Avalos RLM, Rodríguez DSR (1992) Presencia en Veracruz, México, del ácaro Varroa jacobsoni, causante de la varroasis de la abeja melifera (Apis mellifera L.). Técnica Pecuaria en México 30: 133-135.

CONAGUA (2015) Comisión Nacional del Agua. México. http://www. conagua.gob.mx/. Fecha de consulta: 23 Jun 2015.

De Jong D, Roma DA, Goncalves LS (1982) Weight loss and other damage to developing worker honey bees from infestation with a comparative analysis of shaking solutions for the detection of Varroa jacobsoni on adult honeybees. Apidologie 13: 297-306.

Giacobino A, Pacini A, Molineri A, Bulacio N C, Merke J, Orellano E, et al. (2017) Environment or beekeeping management: What explains better the prevalence of honey bee colonies with high levels of Varroa destructor? Research in Veterinary Science 112: 1-6.

Gobierno del Estado de Jalisco (2012) Plan Regional de Desarrollo Región 05 Sureste de Jalisco. https://transparencia.info.jalisco.gob.mx/sites/.../05.\%20Regi\%C3\%B3n\%2005\%20Sureste.pd. Fecha de consulta: 19 de junio de 2017.

Guzman-Novoa E (2016) Colony collapse disorder and other threats to honey bees. In: Cork S, Hall DC, Liljebjelke $\mathrm{K}(\mathrm{ed})$. One health case studies: Addressing complex problems in a changing world. 5M Publishing. Sheffielf, UK. pp: 204-216.

Guzman-Novoa E, Correa-Benítez A, Guzman G, Espinoza-Montaño LG (2011) Colonization, impact and control of Africanized honey bees in Mexico. Veterinaria Mexico 42: 149-178.

Guzmán-Novoa E, Eccles L, Calvete Y, Mcgowan J, Kelly PG, Correa-Benítez A (2010) Varroa destructor is the main culprit for the death and reduced populations of overwintered honeybee (Apis mellifera) colonies in Ontario, Canada. Apidologie 41: 443-450.

Guzman-Novoa E, Hamiduzzaman MM, Espinosa-Montaño LG, Correa-Benítez A, Anguiano-Baez R, PonceVázquez $\mathrm{R}$ (2012) First detection of four viruses in honey bee (Apis mellifera) workers with and without deformed wings and Varroa destructor in Mexico. Journal of Apicultural Research 51: 342-346.

Harris JW, Harbo JR, Villa JD, Danka RG (2003) Variable population growth of Varroa destructor (Mesostigmata: Varroidae) in colonies of honey bees (Hymenoptera: Apidae) during a 10-year period. Environmental Entomology 32: 1305-1312.

Hou CS, Li BB, Deng S, Diao QY (2016) Effects of Varroa destructor on temperature and humidity conditions and expression of energy metabolism genes in infested honeybee colonies. Genetics and Molecular Research 15(3). Doi: 10.4238/gmr.15038997. 
INEGI (2013) Gobierno del estado de Jalisco, Sayula Jalisco. https://www.jalisco.gob.mx/jalisco/municipios. Fecha de consulta: 19 de junio de 2017.

INEGI (2015) Cuéntame de México. http://cuentame.inegi.org.mx/monografias/informacion/jal/territorio/clima. aspx?tema=me. Fecha de consulta: 19 de junio de 2017.

Koleoglu G, Goodwin PH, Reyes-Quintana M, Hamiduzzaman MM, Guzman-Novoa E (2017) Effect of Varroa destructor, wounding and varroa homogenate on gene expression in brood and adult honey bees. PLoS One 12(1) Doi: 10.1371/journal.pone.0169669: e0169669.

Koleoglu G, Goodwin PH, Reyes-Quintana M, Hamiduzzaman MM, Guzman-Novoa E (2018) Varroa destructor parasitism reduces hemocyte concentrations and prophenol oxidase gene expression in bees from two populations. Parasitology Research 117: 1175-1183.

Martínez-Cesareo M, Rosas-Cordoba J, Prieto-Merlos D, Carmona-Gasca A, Peña-Parra V, Avila-Ramos $\mathrm{F}$ (2016) Presence of Varroa destructor, Nosema apis and Acarapis woodi in honey bees (Apis mellifera) of the east region in the State of Mexico. Abanico Veterinario 6: 30-38.

Martínez-Puc JF, Medina LM, Catzín-Ventura GA (2011) Frecuencia de Varroa destructor, Nosema apis y Acarapis woodi en colonias manejadas y enjambres silvestres de abejas (Apis mellifera) en Mérida, Yucatán, México. Revista mexicana de Ciencias Pecuarias 2: 25-38.

Medina LM (1998) Frequency and infestation levels of the mite Varroa jacobsoni Oud. In managed honey bee (Apis mellifera) colonies in Yucatan, Mexico. American Bee Journal 138: 15-127.

Medina LM, Martin SA (1999) A comparative study of Varroa jacobsoni reproduction in worker cells of honey bees (Apis mellifera) in England and Africanized bees in Yucatan, Mexico. Experimental and Applied Acarology 23: 659-667.

Medina-Flores CA, Guzman-Novoa E, Hamiduzzaman MM, Aréchiga-Flores CF, López-Carlos MA (2014a) Africanized honey bees (Apis mellifera) have low infestation levels of the mite Varroa destructor in different ecological regions in Mexico. Genetics and Molecular Research 13: 7282-7293.

Medina-Flores CA, Guzman-Novoa E, Espinosa-Montaño LG, Uribe-Rubio JL, Gutierrez-Luna R, Gutierrez-Piña FJ (2014b) Frequency of varroosis and nosemosis in honey bee (Apis mellifera) colonies in the state of Zacatecas, Mexico. Revista Chapingo Serie Ciencias Forestales y del Ambiente 20: 159-167.

Medina-Flores CA, Esquivel-Marín NH, Lopez-Carlos M, Medina-Cuellar SE, Aguilera-Soto Jl (2018) Estimation of the loss of honey bee colonies in the altiplano and northern regions of Mexico. Ecosistemas y Recursos Agropecuarios 5: 365-371.

Medina-Flores CA, Guzmán-Novoa E, Aréchiga-Flores CF, Aguilera-Soto JI, Gutiérrez-Piña FJ (2011) Efecto del nivel de infestación de Varroa destructor sobre la producción de miel de colonias de Apis mellifera en el altiplano semiárido de México. Revista Mexicana de Ciencias Pecuarias 2: 313-317.

Meixner MD, Kryger P, Costa C (2015) Effects of genotype, environment, and their interactions on honey bee health in Europe Current Opinion. Insect Science 10: 177-184.

Mitton GA, Szawarski N, Ramos F, Fuselli S, Meroi AFR, Eguaras MJ, et al. (2018) Varroa destructor: when reversion to coumaphos resistance does not happen. Journal of Apicultural Research 5: 536-540.

Mondragon L, Spivak M, Vandame R (2005) A multifactorial study of the resistance of honeybees Apis mellifera to the mite Varroa destructor over one year in Mexico. Apidologie 36: 345-358.

Nazzi F, Le Conte Y (2016). Ecology of Varroa destructor, the major ectoparasite of the Western honey bee, Apis mellifera. Annual Review of Entomology 61: 417-432. 
Santillán-Galicia MT, Ball BV, Clark S J, Alderson PG (2010) Transmission of deformed wing virus and slow paralysis to adult bees (Apis mellifera L.) by Varroa destructor. Journal of Apicultural Research and Bee World 89: 141-148.

SARH (1994) Norma official Mexicana NOM-001-ZOO-1994, campaña nacional contra la varroasis de las abejas. Secretaría de Agricultura y Recursos Hidráulicos http://sagarpa.gob.mx/ganaderia/Paginas/Legislacion. aspx. Fecha de consulta: 19 de junio de 2017.

Tarek H, Hamiduzzaman M M, Morfin N, Guzman-Novoa E (2018) Sub-lethal doses of neonicotinoids and carbamate insecticides reduce the lifespan and alter the expression of immune, health and detoxification related genes in honey bees (Apis mellifera). Genetics and Molecular Research 17(2) Doi: 10.4238/gmr16039908. 
